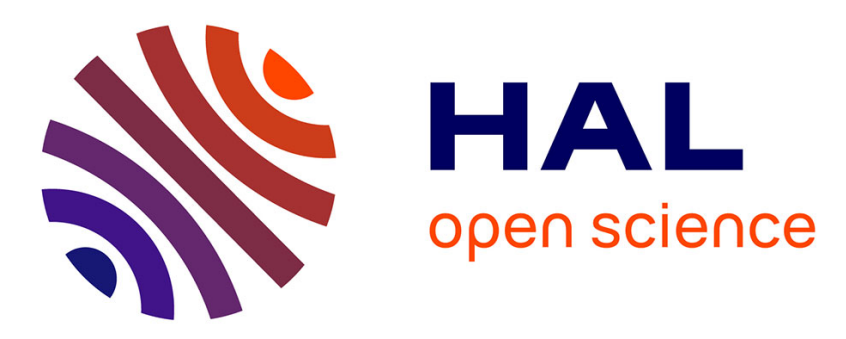

\title{
Detailed modelling of a moving heat source using multigrid methods
}

H. Boffy, Marie-Christine Baietto, Philippe Sainsot, A.A. Lubrecht

\section{To cite this version:}

H. Boffy, Marie-Christine Baietto, Philippe Sainsot, A.A. Lubrecht. Detailed modelling of a moving heat source using multigrid methods. Tribology International, 2012, 46 (1), pp.279-287. 10.1016/j.triboint.2011.06.011 . hal-00797421

\section{HAL Id: hal-00797421 \\ https://hal.science/hal-00797421}

Submitted on 6 Mar 2013

HAL is a multi-disciplinary open access archive for the deposit and dissemination of scientific research documents, whether they are published or not. The documents may come from teaching and research institutions in France or abroad, or from public or private research centers.
L'archive ouverte pluridisciplinaire HAL, est destinée au dépôt et à la diffusion de documents scientifiques de niveau recherche, publiés ou non, émanant des établissements d'enseignement et de recherche français ou étrangers, des laboratoires publics ou privés. 


\title{
Detailed modelling of a moving heat source using multigrid methods
}

\author{
H. Boffy ${ }^{\mathrm{a}}$, M.C. Baietto ${ }^{\mathrm{a}}$, P. Sainsot ${ }^{\mathrm{a}}$, A.A. Lubrecht ${ }^{\mathrm{a}}$, \\ ${ }^{\text {a }}$ Université de Lyon, INSA-Lyon, LaMCoS, CNRS UMR 5259, Villeurbanne F69621, France
}

The actual contact between solid surfaces is generaly rough and time dependent. This roughness can only be correctly described using a 3d model. Moreover, severe thermo-mechanical loading induces high stress and temperature gradients within a thin subsurface layer. Even higher gradients are obtained in the case of surface coatings. Consequently, the problem is strongly multiscale: from contact to coating to roughness, the characteristic dimensions range from millimeter to nanometer.

A straightforward discretisation of this multiscale problem would exceed the memory and CPU capabilities of current computers. The aim of this work is to propose a more efficient numerical model able to deal with this multiscale problem: using $10^{9}$ points and $10^{3}$ timesteps.

This paper traces the history of the numerical solutions of the heat equation from the pioneering work of Carslaw and Jaeger, to the current era.

The proposed model is based on multigrid techniques within a finite difference frame work. Localised refinement is implemented to optimize memory and computing time costs. The numerical performance of the solver is presented through a comparison with analytical results using different types of boundary conditions. A multisource contact is studied as a first approximation to real asperity interaction.

\section{Introduction}

Stationary and moving heat source problems are frequently encountered in tribological applications [1] (frictional heating) as well as in many manufacturing processes [2] (metal cuting, laser treatment). Moreover, these problems which are complex and multiscale (time dependent, moving component, heat source distribution [3], dimensions ranging over several decades and boundary conditions) are at the root of different types of mechanical failure [4].

Many researchers have studied frictional heating. Some have analyzed a semi-infinite thermally isolated solid subjected to a heat source [5][6][7], or to several sources [8]. More recently, a relativistic moving heat source has been studied [9].

This paper proposes a multigrid solution method to deal with the transient thermal problem. The proposed method is fast and particularly flexible compared with other methods (FFT,FEM). The direct solution of the heat equation allows one to deal with any kind of source displacement and speed. Multigrid methods enable general source geometries as their efficiency permits thousands of points in the contact area. Moreover, these methods allow efficient solutions, especially when including a local refinement strategy. Good convergence rates are obtained using single-grid calculations when considering Dirichlet boundary conditions. Local mesh refinement can be used [10]. However, very fine grids are often needed to capture thin boundary layer properties and to detect heat transfer spots. A local refinement strategy allows one to reduce the calculation time and use an adaptive mesh size where gradients are largest. The influence of the Peclet number has also been analyzed [11]. 


\section{$2 \quad 3 \mathrm{D}$ Thermal Model}

\subsection{Thermal equation}

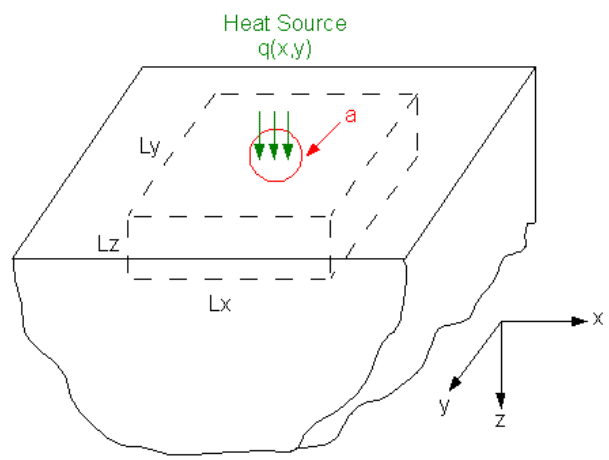

Figure 1: Description of the physical domain and coordinate system.

The differential equation of heat conduction for a stationary, homogeneous, isotropic semi-infinite solid (cf fig1) with constant thermal properties and no heat generation reads (Fourier conduction equation):

$$
\frac{\partial^{2} T}{\partial x^{2}}+\frac{\partial^{2} T}{\partial y^{2}}+\frac{\partial^{2} T}{\partial z^{2}}=\frac{1}{D} \frac{\partial T}{\partial t}
$$

where $\mathrm{D}$ is the thermal diffusivity.

Using the following dimensionless parameters:

$$
X=\frac{x}{a} ; Y=\frac{y}{a} ; Z=\frac{z}{a} ; \bar{t}=\frac{t}{\tau} ; \bar{T}=\frac{T}{\Delta T} ; \tau=\frac{a^{2}}{D}
$$

Where $a$ is the characteristic dimension in $x, y$ and $z . \tau$ is the characteristic time. $\Delta T$ corresponds to the temperature increase compared with room temperature. The reduced form of equation (1) becomes:

$$
\frac{\partial^{2} \bar{T}}{\partial X^{2}}+\frac{\partial^{2} \bar{T}}{\partial Y^{2}}+\frac{\partial^{2} \bar{T}}{\partial Z^{2}}=\frac{\partial \bar{T}}{\partial \bar{t}}
$$

The thermal boundary conditions (BC) are either: a prescribed temperature derivative (Neumann BC) or an imposed temperature value (Dirichlet BC). On the surface boundary $(z=0)$ the heat flux is prescribed:

$$
q(x, y, t)=-\left.k \frac{\partial T}{\partial z}\right|_{z=0}
$$

Using the following dimensionless parameters:

$$
\begin{aligned}
Q=\frac{q}{q_{\max }} & =-\frac{k}{q_{\max }} \frac{\Delta T}{a} \frac{\partial \bar{T}}{\partial \bar{Z}} \\
\Delta T & =\frac{a q_{\max }}{k}
\end{aligned}
$$

One obtains: 


$$
Q=-\frac{\partial \bar{T}}{\partial \bar{Z}}
$$

On all other boundaries the temperature is imposed.

\subsection{Discretization}

The partial differential equation PDE is discretised using finite difference techniques. This discrete approximation introduces an error with respect to the solution of the PDE. A discretised first order accurate approximation for the Poisson equation and the time derivate is:

$$
\frac{\bar{T}_{i-1, j, k}-2 \bar{T}_{i, j, k}+\bar{T}_{i+1, j, k}}{h_{x}^{2}}+\frac{\bar{T}_{i, j-1, k}-2 \bar{T}_{i, j, k}+\bar{T}_{i, j+1, k}}{h_{y}^{2}}+\frac{\bar{T}_{i, j, k-1}-2 \bar{T}_{i, j, k}+\bar{T}_{i, j, k+1}}{h_{z}^{2}}=\frac{\bar{T}_{i, j, k}-\bar{T} O_{i, j, k}}{h_{t}}
$$

$h_{x}, h_{y}$ and $h_{z}$ are the mesh-size in $x, y$ and $z$-direction, respectively, $\bar{T}_{i, j, k}$ is the discrete approximation of the unknown $T\left(x_{i}, y_{j}, z_{k}\right)$ to be solved and $\bar{T} O_{i, j, k}$ is the discrete representation of $T\left(x_{i}, y_{j}, z_{k}\right)$ at time t-1. To simplify the notation a stencil is introduced. Using this stencil $\mathcal{L}$ equation $(8)$ is written as:

$$
L<T>_{i, j, k}=\frac{-\bar{T} O_{i, j, k}}{h_{t}}
$$

with :

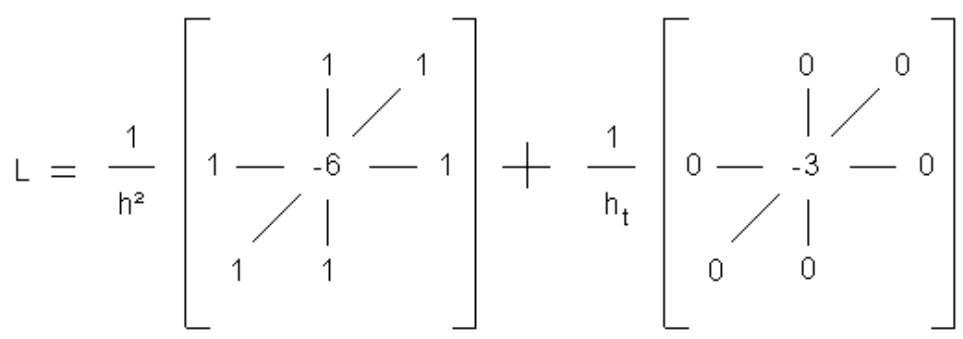

Figure 2: Heat transfer stencil.

where the gridsize is assumed to be equal in each direction $\left(h_{x}=h_{y}=h_{z}=h\right)$.

At the surface, both Dirichlet and Neumann conditions can be implemented. In the case of Neumann BC, one uses a second-order discretization leading to:

$$
\bar{T}_{i, j, k-1}=\bar{T}_{i, j, k+1}-2 h Q
$$

The unknown $\bar{T}_{i, j, k-1}$ is then substituted in the discretized heat equation at the surface $(z=0, k=0)$.

\section{Local MultiGrid solver}

The heat problem is solved as a linear system of the form:

$$
\mathcal{L} \bar{T}=f
$$

Where $\mathcal{L}$ is a differential operator, $\bar{T}$ the exact solution of the problem and $f$ the right hand side of the equation. Multigrid methods, employed to solve this problem, have been pioneered by Brandt [16]. Further details concerning the implementation can be found in Venner-Lubrecht [17]. 


\subsection{Boundary conditions}

Solving the thermal problem considering Neumann BC adds several difficulties. In order to obtain a solver with the same efficiency as the one employing Dirichlet BC, the BC have to fulfill two constraints: consistency on various grids, no local error introduction.

Venner et al. [17] relax the interior problem on the surface using the Neumann BC to fulfill both criteria. fig.(3) shows the results obtained: the convergence speed is not affected by the type of boundary condition but the residual is higher for the Neumann case because the solver does not start with the solution on the surface. Some local prerelaxations are necessary to decrease the residual near the boundaries before solving the problem on the entire grid.

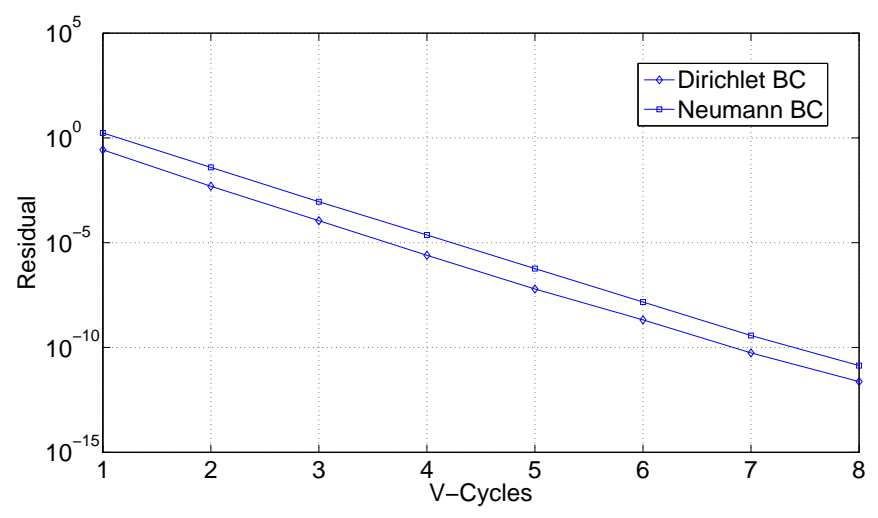

Figure 3: Residual reduction on the finest level as a function of the number of V-cycles for Dirichlet and Neumann BC.

\subsection{Mesh refinement strategy}

The solution of a fast moving heat source requires a multiscale approach, both in space and time. Dimensions ranging from microns to meters are concerned: a near subsurface layer $(\mu m)$, a loaded zone $(\mathrm{mm})$, specific volume dimensions $(x, y, z)(\mathrm{cm}, \mathrm{m})$. Furthermore, depending on the Peclet number, the time step can range from microseconds to seconds. A very fine grid is required around the heat source to capture the large gradients. Elsewhere such level of refinement is not required, especially when considering a semiinfinite solid. MG methods are very convenient for local mesh refinement. The finest grids can be restricted to smaller and smaller subdomains, whereas the coarse grids cover the entire domain (fig.(4b)). The fine grid residuals and the coarse grid correction are only carried out in the local part where the finest grid exists. The unit disk flow problem is solved using 6 levels, involving a coarsest grid with $16^{*} 16^{*} 8$ and a finest grid of $512^{*} 512^{*} 256$ points. The computational domain is $X \in[-8,8], Y \in[-8,8], Z \in[-8,0]$. Local refinement does not affect the convergence speed as shown in Fig.4(a). The convergence rate in both cases is approximately the same.

\section{Validation}

\subsection{Green's function}

To validate the numerical model, cases with analytical solutions have been computed. The general solution for the heat equation with a moving source in $X$ direction $\left(P e=U_{s} \tau / a\right)$ is given by Jaeger [13] : 

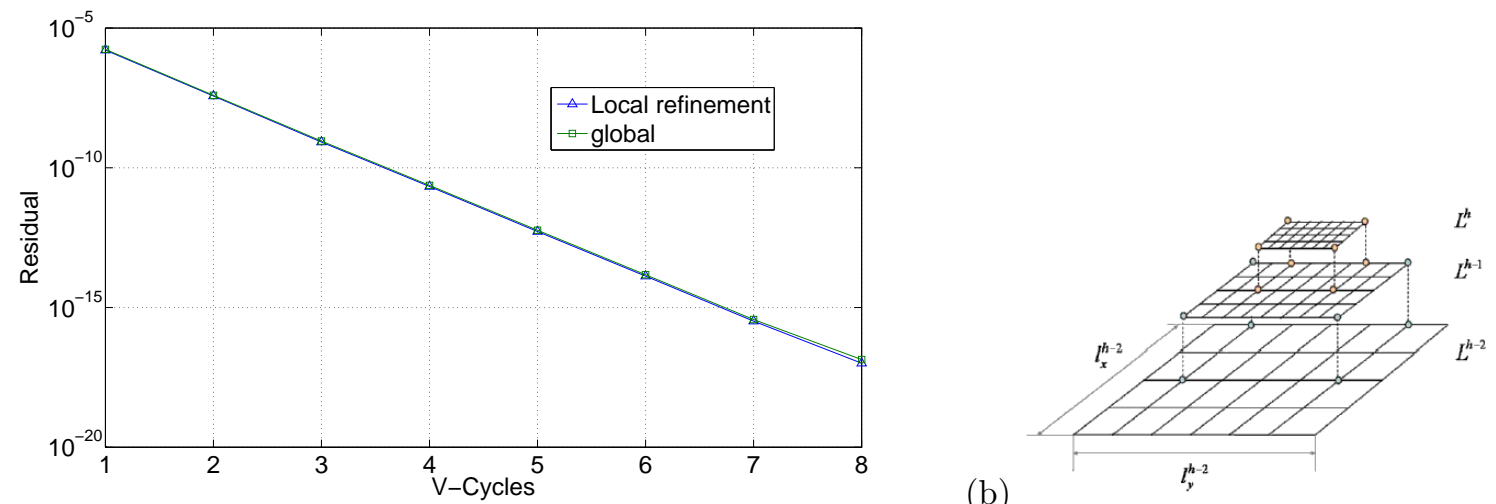

Figure 4: (a) Global and Local residual reduction obtained for the case of a unit disk flow (b). Local 2-D grids

$\bar{T}(X, Y, Z, \bar{t})=\int_{0}^{\bar{t}} \frac{2}{\left[4 \pi\left(\bar{t}-\bar{t}^{\prime}\right)\right]^{3 / 2}} \iint Q\left(X^{\prime}, Y^{\prime}, \overline{t^{\prime}}\right) \exp \left[-\frac{\left(X-X^{\prime}-\left[\int_{\bar{t}^{\prime}}^{\bar{t}} P e(\bar{\tau}) d \bar{\tau}\right]\right)^{2}+\left(Y-Y^{\prime}\right)^{2}+Z^{2}}{4\left(\bar{t}^{\prime}-\bar{t}^{\prime}\right)}\right] d X^{\prime} d Y^{\prime} d \overline{t^{\prime}}$

For a stationary flux one obtains :

$$
\bar{T}(X, Y, Z, \bar{t})=Q(X, Y) * \int_{0}^{\bar{t}} G\left(X, Y, Z, \bar{t}-\bar{t}^{\prime}\right) d \bar{t}^{\prime}
$$

Liu, Lannou et al. [15] give an algebraic solution for the time integrated Green's function for a constant speed $(P e=$ constant $)$ :

$\int_{0}^{\bar{t}} G\left(X, Y, Z, \bar{t}-\bar{t}^{\prime}\right) \overline{d t^{\prime}}=G^{s}(X, Y, Z, \bar{t})=\frac{e^{-P e(R-X) / 2}}{4 \pi R}\left[2-\operatorname{erfc}\left(\frac{P e \sqrt{\bar{t}}}{2}-\frac{R}{2 \sqrt{t}}\right)+e^{(R * P e)} \operatorname{erfc}\left(\frac{P e \sqrt{\bar{t}}}{2}+\frac{R}{2 \sqrt{\bar{t}}}\right)\right]$

With $R^{2}=X^{2}+Y^{2}+Z^{2}$

\subsection{Stationary case}

\subsubsection{Analytical solution}

For a stationary source and established solution, i.e. $P e=0$ and $\bar{t}=\infty$, one obtains:

$$
\int_{0}^{\infty} G\left(X, Y, Z, \bar{t}-\bar{t}^{\prime}\right) \overline{d t}^{\prime}=G^{P e}(X, Y, Z)=\frac{1}{2 \pi R}
$$

The same Green's function is found for a normal displacement due to a normal strain in solid mechanics. Four analytical cases are presented: constant flux on a square surface, constant flux on a disk surface, inverse elliptical flux on a disk surface, hertzian flux on a disk surface.

- For a constant flux applied on the surface of a unit square, i.e. $Q(X, Y, Z=0, \bar{t})=1$, one obtains: 
$T(X, Y, Z=0)=\frac{1}{2 \pi}\left[\left[X_{i} * \log \left(Y_{i}+\sqrt{X_{i}^{2}+Y_{i}^{2}}\right)+Y_{i} * \log \left(X_{i}+\sqrt{X_{i}^{2}+Y i^{2}}\right)\right]_{X-d x / 2}^{X+d x / 2}\right]_{Y-d y / 2}^{Y+d y / 2}$

$T(0,0,0)=2 * \ln (1+\sqrt{2}) / \pi$

This solution is shown in fig.5.(a).

- For $Q(X, Y, Z=0)=1$ on a disk with a radius $R_{a y}$ centered on O. One obtains :

if $R<R_{a y}$

$$
\begin{gathered}
T(X, Y, Z=0)=R_{a y} * \frac{2}{\pi} * E\left[\frac{R}{R_{a y}}\right] \\
R_{a y}=1 ; \quad T(0,0,0)=1 ; \quad T\left(R=R_{a y}, 0\right)=\frac{2}{\pi}
\end{gathered}
$$

if $R>R_{a y}$

$$
T(X, Y, Z=0)=R_{a y} * \frac{2}{\pi} * R\left[E\left[\frac{R}{R_{a y}}\right]-\left(1-\frac{R_{a y}^{2}}{R^{2}}\right) * K\left[\frac{R_{a y}}{R}\right]\right]
$$

With $E$ the Young modulus and $K=E / 2(1+\nu), \nu$ the poisson coefficient. The solution is shown in fig.5.(b).

- For $\mathrm{Q}(\mathrm{X}, \mathrm{Y})=1 / \sqrt{1-\left[R / R_{a y}\right]^{2}}$ on a disk with a radius $R_{a y}$ centered on O. Calculations give:

if $R<R_{a y}$

$$
\begin{gathered}
T(X, Y, Z=0)=R_{a y} * \frac{\pi}{2} \\
T(R=0,0)=R_{a y} * \frac{\pi}{2} ; \quad T\left(R=R_{a y}, 0\right)=R_{a y} * \frac{\pi}{2}
\end{gathered}
$$

if $R>R_{a y}$

$$
T(X, Y, Z=0)=R_{a y} * \arcsin \left(\frac{R_{a y}}{R}\right)
$$

The solution is shown in fig.5.(c).

- For $\mathrm{Q}(\mathrm{X}, \mathrm{Y})=\sqrt{1-\left[R / R_{a y}\right]^{2}}$ on a disk with a radius $R_{a y}$ centered on O. Calculations give: if $R<R_{a y}$

if $R>R_{a y}$

$$
\begin{gathered}
T(X, Y, Z=0)=R_{a y} * \frac{\pi}{8}\left(2-\frac{R^{2}}{R_{a y}^{2}}\right) \\
T(R=0,0)=R_{a y} * \frac{\pi}{4} ; \quad T\left(R=R_{a y}, 0\right)=R_{a y} * \frac{\pi}{8}
\end{gathered}
$$

$$
T(X, Y, Z=0)=\frac{1}{4} R_{a y}\left[\left(2-\frac{R^{2}}{R_{a y}^{2}}\right) * \arcsin \left(\frac{R_{a y}}{R}\right)+\frac{R}{R_{a y}} \sqrt{1-\frac{R_{a y}^{2}}{R^{2}}}\right]
$$

The solution is shown in fig.(5d). Calculation have been done on a cubic volume which is not a semiinfinite one. Consequently, Dirichlet boundary condition are used on the borders of the volume as given in eq(15) excepted on the surface where a flux is applied. To approximate at best the steady-state results, convergence has been pursued until residuals were less than $10^{-8}$. 


\subsubsection{Numerical solution}

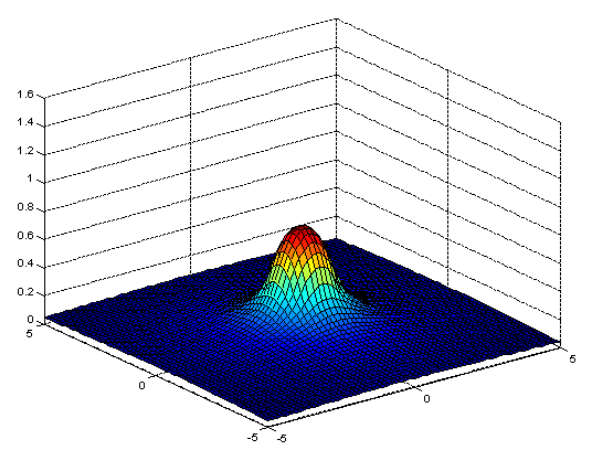

(a)

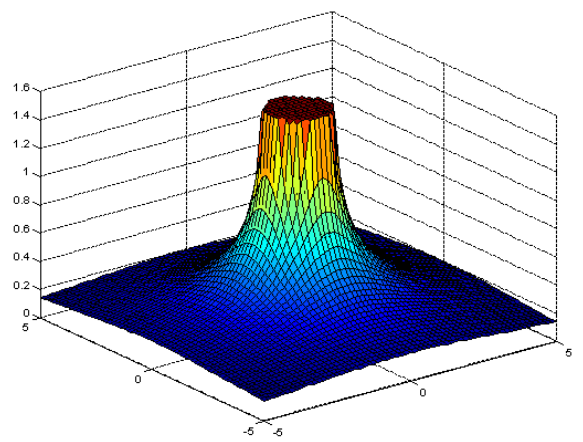

(c) (b)
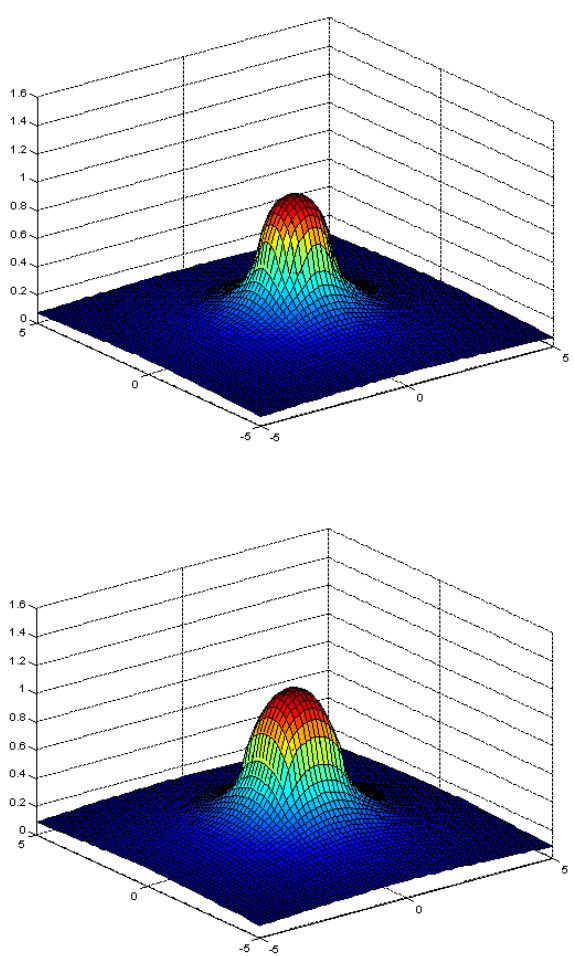

(d)

Figure 5: Numerical temperature distribution for a unit square flux, unit disk flux, disk flux with $\mathrm{Q}(\mathrm{X}, \mathrm{Y})$ $=1 / \sqrt{1-\left[R / R_{a y}\right]^{2}}$ and disk flux with $\mathrm{Q}(\mathrm{X}, \mathrm{Y})=\sqrt{1-\left[R / R_{a y}\right]^{2}}$ left to right, top to bottom

The temperature distributions of equations (16) to (22) are plotted in fig.(5). These thermal problems are numerically solved using the MG technique presented in this paper. In further sections the numerical solutions will be compared with the analytical solutions. The dimensions of the domain with respect to the heat source, the mesh size and consequently the numerical cost are analyzed through a detailed comparison. The discretization error is studied through a convergence analysis. The efficiency of the local refinement strategy is demonstrated through the evolution of the CPU time.

\subsubsection{Effect of boundary condition}

The problem analysed is a unit disk flow applied on the surface (18). The coarsest grid is discretized using $16^{*} 16^{*} 8$ points. In order to approximate the semi-infinite conditions, boundary conditions have been implemented on the borders according to the far field solution (15).

Fig.(6) shows the influence of the domain size on the central temperature for a constant mesh size. The error in the temperature field is caused by the finite dimensions of the volume studied. The order of magnitude of this error has to be evaluated to ensure it does not dominate the overall solution. The next subsection shows how one can increase accuracy by increasing the domain size.

\subsubsection{Effect of mesh size and CPU time}

In this subsection the domain size and the number of grid points in the source are analysed. Two aspects need to be studied, the first one concerns the decrease of the numerical error with the mesh size. The 


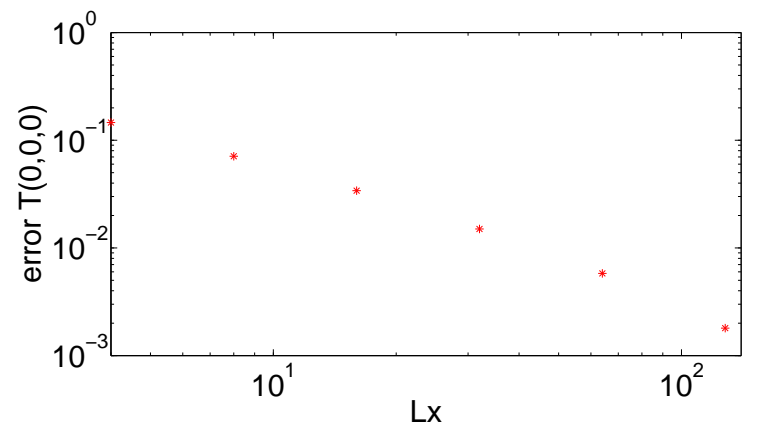

Figure 6: Effect of domain size on $T(0,0,0)$ for a unitary disk flow, for a constant discretization error: 65 points in the source (finest grid)

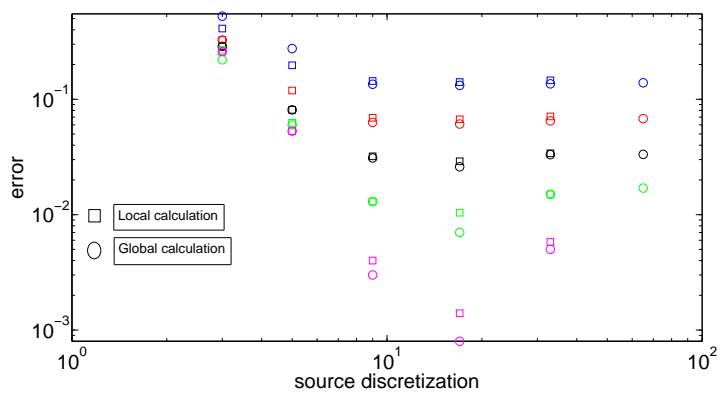

Figure 7: Relative error between analytical and numerical solutions as a function of the source discretization, circular unit flux, $16^{*} 16^{*} 8$ points on the coarse grid. Volume size increase by a factor of 2 from top $(4 * 4 * 2)$ to bottom $(64 * 64 * 32)$. Global and local refinement results

second one concerns the error as a function of the domain size. The error in the central temperature is also plotted in fig.(7) as a function of the source discretization. One can remark a similar error reduction as in fig.(6). Furthermore, the error stagnates for a certain source discretization for each domain size. Increasing the domain allows a further error reduction. This figure also shows the efficiency of the local refinement strategy, which does not significantly affect the precision. Fig.(8) gives the CPU time using global and local calculations, with a residual less than $10^{-12}$. One can conclude that the problem can be solved with almost the same accuracy but a 100 times smaller computational effort using local grid refinement.

\subsection{Moving heat source}

\subsubsection{Analytical solution}

A moving heat source problem can be characterized using the Peclet number, defined by $U_{s}=\tau V / a$. This number is directly linked to the mesh size ( $a$ is the characteristic dimension in $x$ and $y$ direction). A non-dimensional unit velocity corresponds to a single mesh size displacement per time step. A well-known $2 \mathrm{D}$ solution describes a moving heat band on the surface of a semi-infinite volume.

The solution has been calculated by Jaeger [12]. For large values of $\mathrm{Pe}(>10)$, the heat will diffuse only a short distance into the solid in the time taken for the surface to move through the heated zone. The heat flow will then be approximately perpendicular to the surface at all points. The temperature of a surface point is given by Carslaw and Jaeger [13]. 


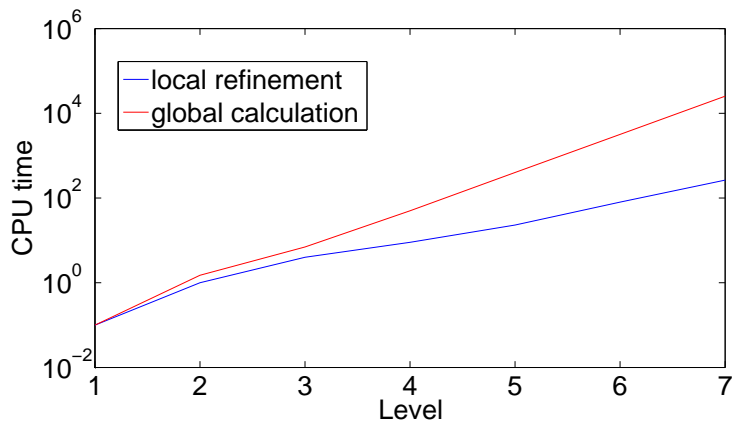

Figure 8: CPU time (in seconds).

$$
\begin{aligned}
& \bar{T}=\left[\frac{4}{\pi P e}(1+X)\right]^{1 / 2} \\
& \bar{T} \sqrt{P e}=\left[\frac{4}{\pi}(1+X)\right]^{1 / 2}
\end{aligned}
$$

With $-1 \leq X \leq 1$

The mean temperature for a band source is approximately:

$$
\bar{T}_{\text {mean }} \approx \frac{4 Q}{3 \pi^{1 / 2}} P e^{-1 / 2}
$$

\subsubsection{Numerical solution}

In this subsection, the problem of the infinitely wide moving source is studied. The source moves in the $x$ direction. The comparison between analytical (23) and numerical solution $(\bar{T} * \sqrt{P e})$ is plotted in fig.(9). The calculations use 3 global and 4 local levels in $y$ and $z$ direction. The band source is discretized using 129 points in the $x$-direction.

Fig.(9.a) shows that for increasing Peclet number, the numerical solution tends to the analytical one. Fig.(9.b) shows the evolution of the error between both solutions. This evolution is approximately first order as can be expected from (8). At very low speeds $(\mathrm{Pe}<0,1)$ the temperature distribution becomes symmetrical and similar to that of a stationary source.

\section{General sources}

In this section, a moving multisource problem is studied to approximate a rough thermal contact. Several circular fluxes are applied on the surface in a circular area, as shown in fig (10). The other boundaries are kept at a prescribed temperature. Gao et al. [18] and Yu et al. [19] studied this kind of problem using the FFT method. The 7 heat spots move with a velocity defined by the Peclet number. In a first subsection the influence of the size of the spots is studied, keeping the Peclet number constant. In a second subsection the effect of speed is studied, looking at several Peclet numbers and keeping the spot size constant. 
(a)

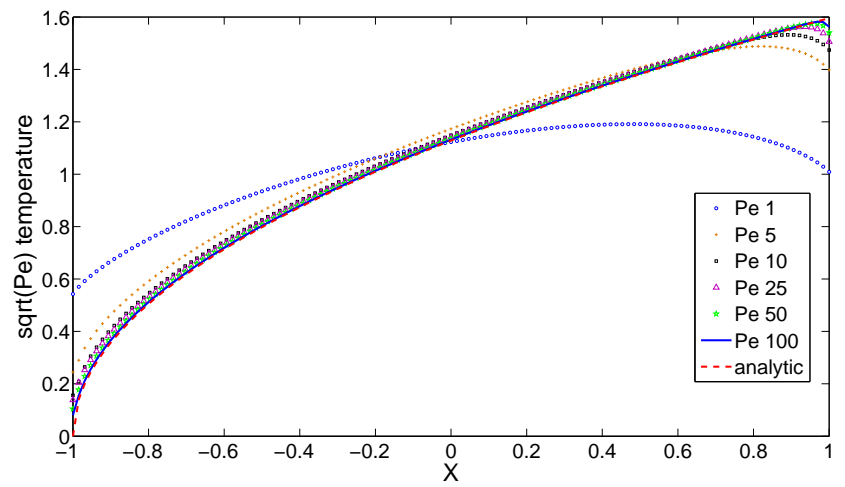

(b)

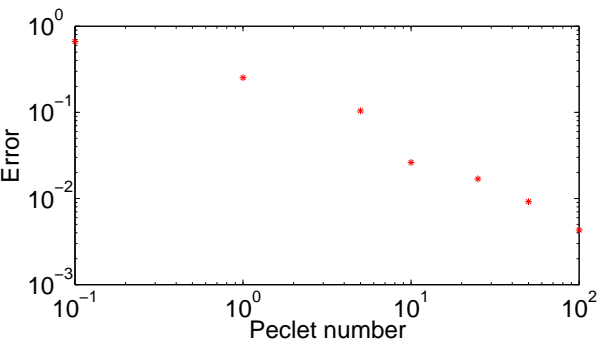

Figure 9: (a)Comparison between analytical and numerical solutions for increasing Peclet number. (b)Error between analytical and numerical solution for $\mathrm{Pe}=1,5,10,25,50$ and 100

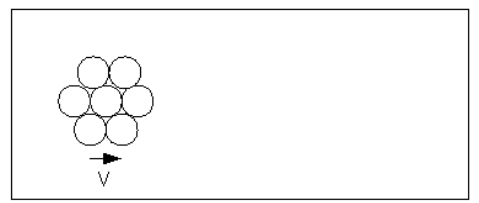

Figure 10: Multisource configuration.

\subsection{Speed effect}

The volume studied has dimensions of $64^{*} 32^{*} 16$, the coarsest grid is discretized using $64^{*} 32^{*} 16$ points. The local refinement strategy is used: the calculations have been performed using 6 or 7 levels (for high Peclet numbers $(>10)$ ): a global one and 5 or 6 local ones. Each small disk is discretized using approximately 30 points on the finest grid. Several Peclet numbers have been used: 1, 2, 10 and 100. In order to visualize the influence of the spot size, 3 diameters have been studied: $1 / 3,1 / 4$ and $1 / 8$. When using a radius of $1 / 3$, the disks are in contact. The overall intensity in each unitary disk is kept constant $(\pi)$. The fig.(11) shows that the global temperature profile (outside the source) is not affected by the source micro-geometry. The following study will consequently look after the temperature distribution inside the source. The surface temperature is plotted in the $x$ direction for each Peclet number. These curves are compared with the smooth unit disk result. The results are shown in fig.(12):

One finds the usual results of a moving heat source. The curves show that each disk flow is little affected by the other ones for a radius less than $1 / 4$. The maximum flash temperature rise [20] is located towards the trailing regions of the contact depending of the Peclet number. In addition, the maximum flash temperature decreases when the Peclet number increases. For high Peclet numbers $(>10)$, the temperature profile in each disk becomes more and more pointed. Flash temperatures are expressed in terms of the heat supply over the contact area, the velocity, and the thermal properties of the material. These have been developed 


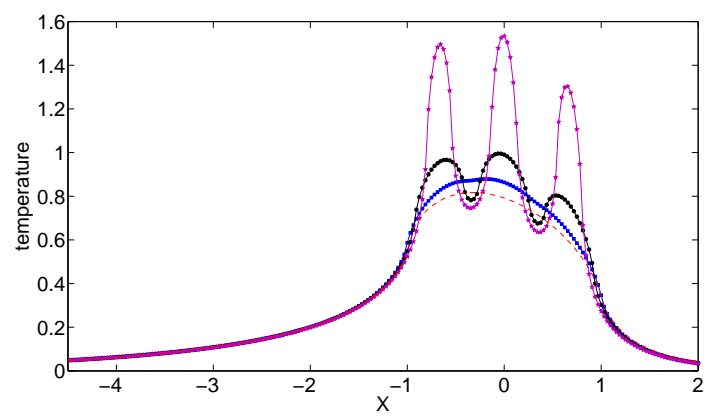

Figure 11: $\mathrm{T}(\mathrm{X}, 0,0)$ for a Peclet number of 1 . Solutions for $\mathrm{r}=1 / 8,1 / 4,1 / 3$ and a unit disk are plotted top to bottom.
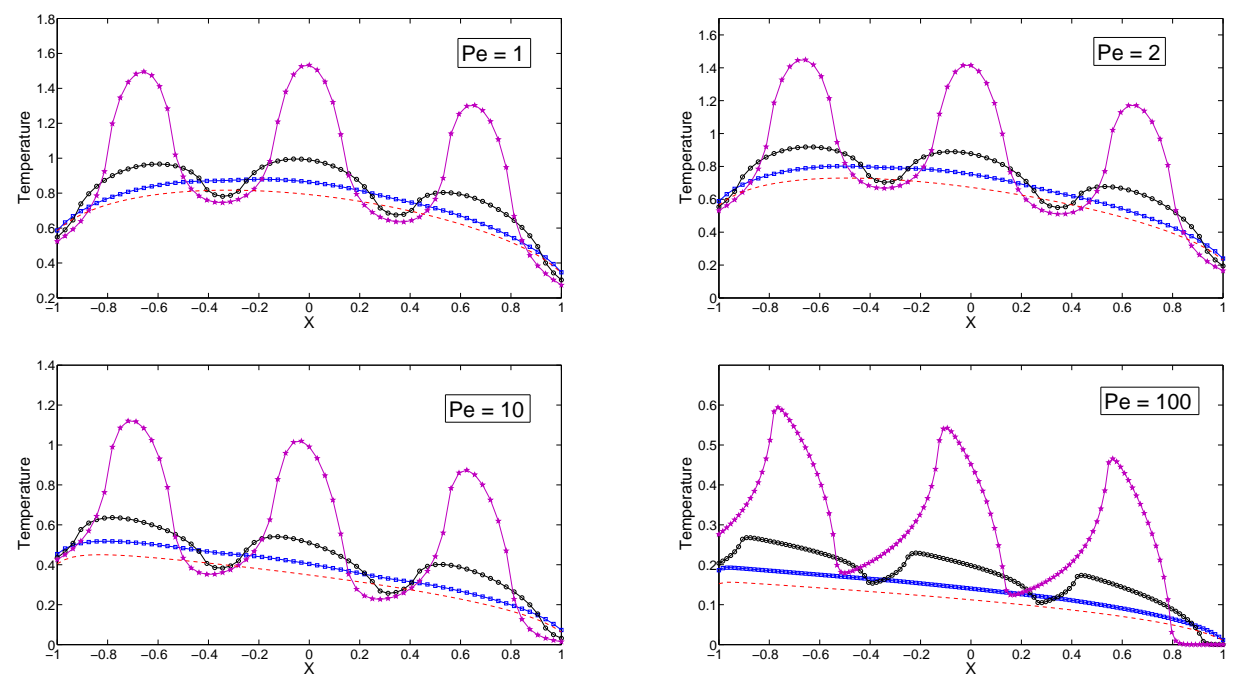

Figure 12: $\mathrm{T}(\mathrm{X}, 0,0)$ for different Peclet numbers with $\mathrm{r}=1 / 8,1 / 4,1 / 3$ and unit disk solution top to bottom.

by Blok and Jaeger for linear and square contacts [21,13] and by Archard [22] for circular contacts.

\section{$5.2 \quad$ Size effect}

In this subsection, the effect of the radius variation is analyzed. The same curves as in fig.12. are presented, showing the same radius in each sub-figure for different Peclet numbers, fig.13.

One remarks that the curves with a radius of $1 / 3$ look like the ones obtained for the semi infinite band case. When the radius decreases, the flash temperatures are better distinguished. The profiles obtained can be considered as a first approximation of a rough contact between two solids.

\subsection{Sub-layer analysis}

In this subsection, the temperature field below the heat source is analyzed. The objective is to see how velocity affects the temperature in the sublayer. Fig.14-16. shows the temperature fields for the same Peclet numbers and radii as in fig. 11 and 12.

The temperature field for a Peclet number less than 0.2 (not shown) closely resembles the stationary case [12]. For medium Peclet numbers (0.2 to 10), the temperature distribution remains largely symmetrical. 

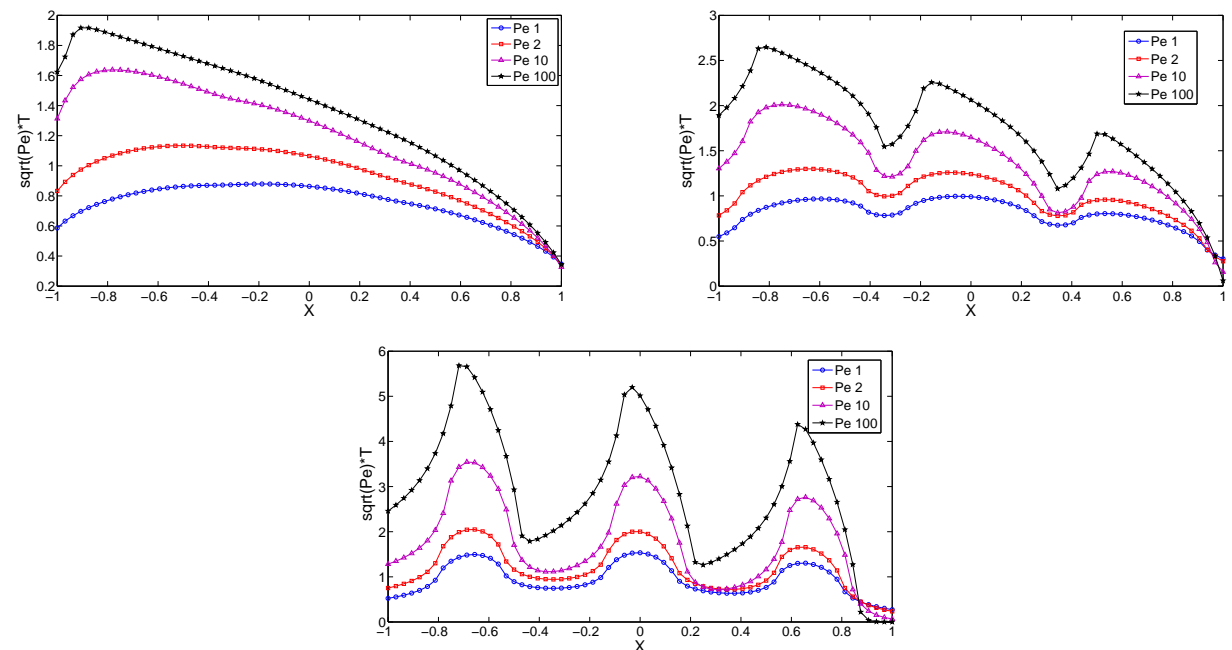

Figure 13: $\bar{T}(\mathrm{X}, 0,0) * \sqrt{P e}$ for different source radii with $\mathrm{Pe}=100,10,2,1$ top to bottom
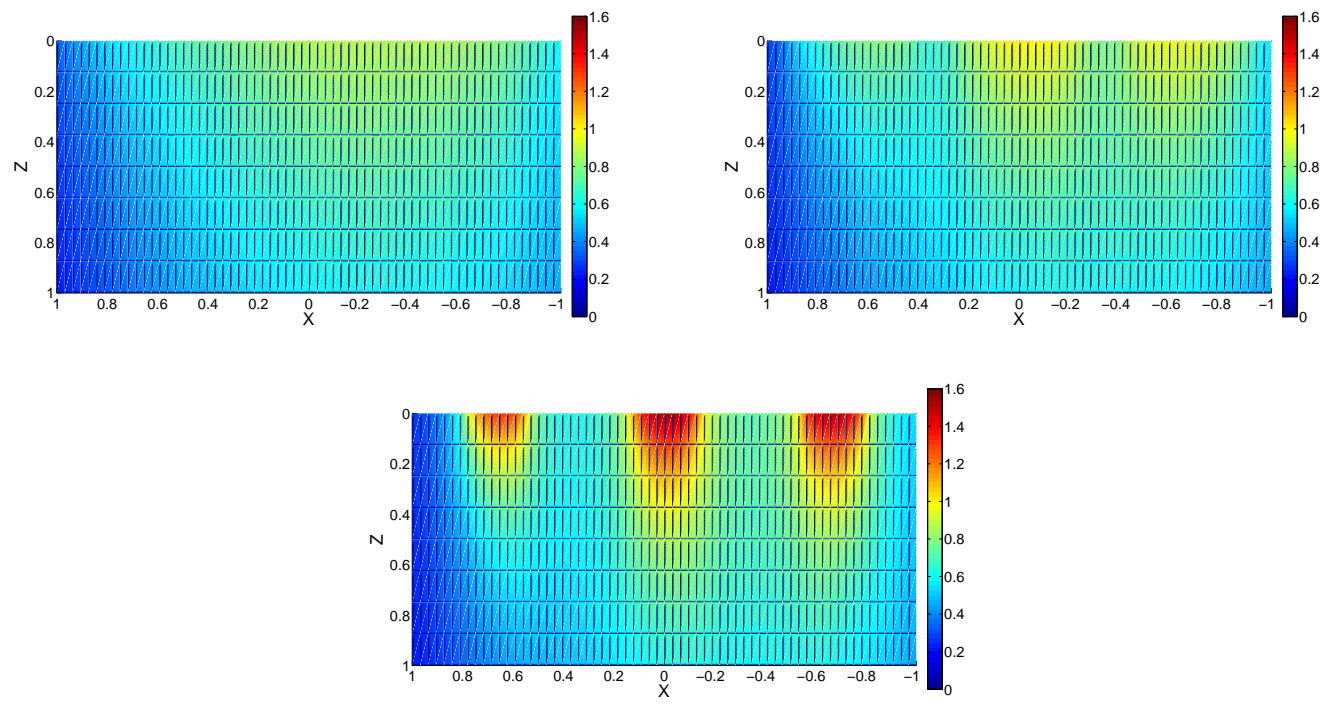

Figure 14: $T(X, Y=0, Z)$ for a Peclet number of $1, r=1 / 3,1 / 4,1 / 8$

The temperature influence occurs closer to the surface when speed increases [23].

\section{Conclusion and further research}

A 3-D model is presented to solve the heat equation. This model can handle multiple moving sources using multigrid techniques which enable fine mesh sizes and local refinement techniques. These methods keep calculation time acceptable on desktop computers. Multigrid techniques and local refinement greatly reduce the calculation time. Macroscopic and microscopic aspects have been studied: the importance of 

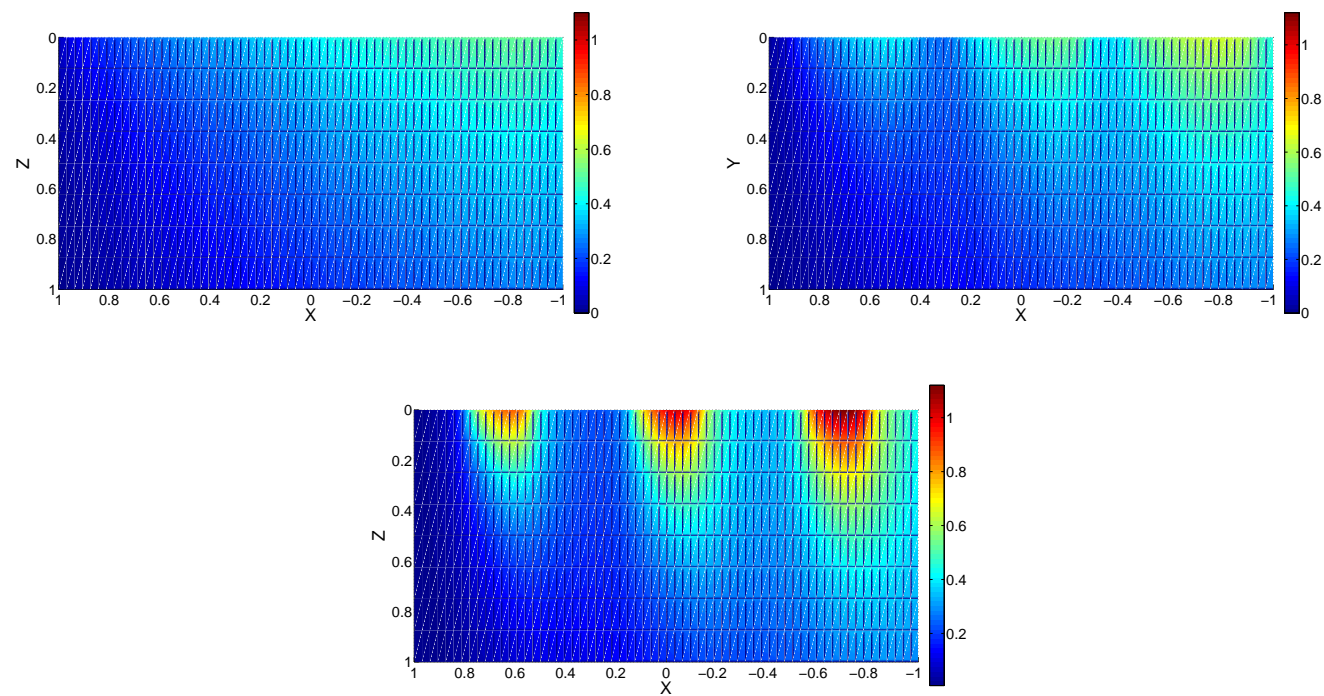

Figure 15: $T(X, Y=0, Z)$ for a Peclet number of $10, r=1 / 3,1 / 4,1 / 8$
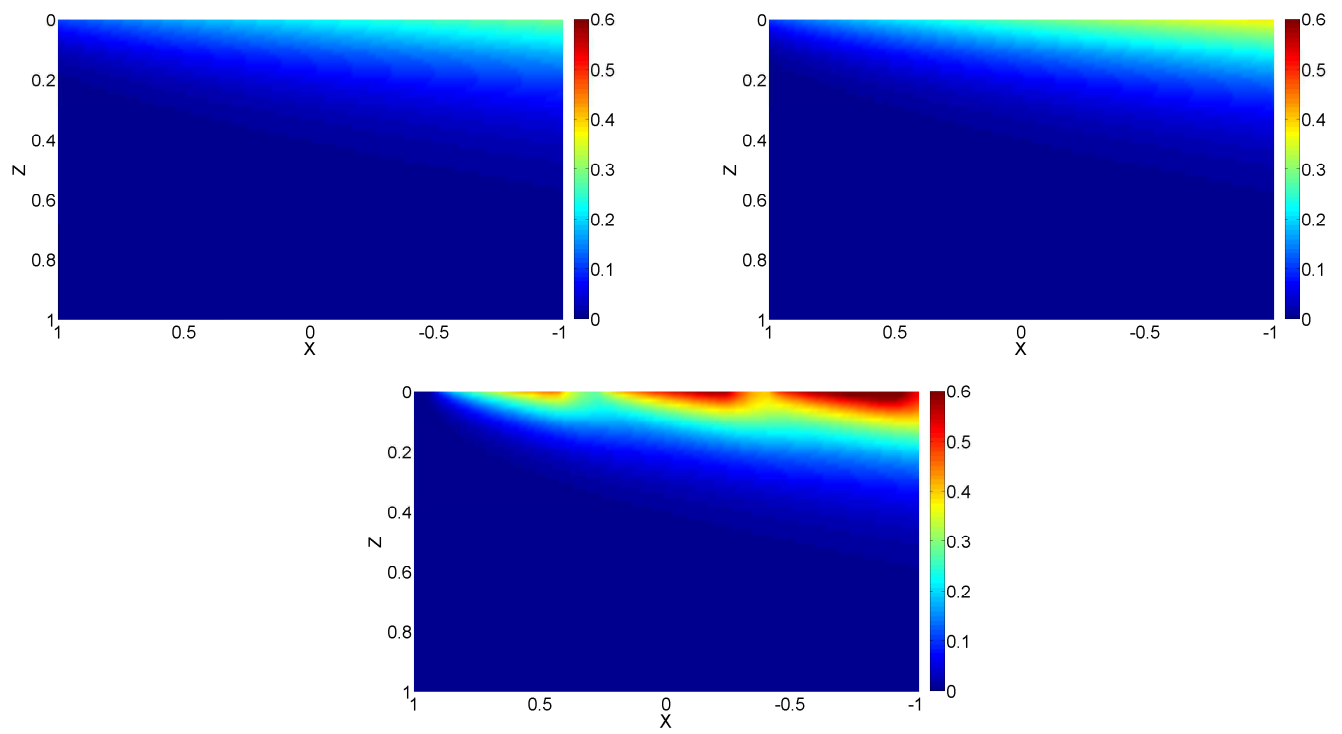

Figure 16: $T(X, Y=0, Z)$ for a Peclet number of $100, r=1 / 3,1 / 4,1 / 8$

the macroscopic aspect was demonstrated through the influence of the domain size. The borders of the volume have to be distant enough from the source to respect the semi-infinite conditions. Concerning the microscopic aspects, it has been shown that the refinement strategy allows a good accuracy and permits one to observe microscopic phenomena close to the source. Using the present code, one can obtain an accuracy better than $10^{-2}$ as shown by the comparison between computed and analytical solutions. The algorithm reaches this accuracy because the hypothesis of a semi-infinite volume has been closely respected using a large enough volume. Moreover, calculations can be performed using $10^{9}$ points on the finest grid. Furthermore stationary and moving sources, single and multiple sources have been studied. The increase in velocity creates a reduction in the temperature amplitude and a displacement of the temperature maximum 
in the direction of the movement. Our major objective is to extend this work towards 3D thermoelastic calculations.

\section{References}

[1] Cowan, R. S., and Winer, W. O., 1992, "Frictional Heating Calculations," ASM Handbook, Vol. 18: Friction, Lubrication, and Wear Technology, ASM International, pp. 39-44.

[2] Komanduri, R., and Hou, Z. B., 2001, "Thermal Modeling of the Metal Cuting Process, Part II Temperature Rise Distribution Due to Frictional Heat Source at the Tool-Chip Interface," Int. J. Mech. Sci., 43, pp.57-88.

[3] Hou, Z.B., and Komanduri, R., 2000, "General Solutions for Stationary/Moving Plane Heat Source Problems in Manufacturing and Tribology," Int. J. Heat Mass Transfer, 43, pp. 1679-1698.

[4] Kennedy, F. E., 1984, "Thermal and Thermomechanical Effects in Dry Sliding," Wear, 100, pp.453-476.

[5] Jaeger, J.C., "Moving sources of heat and the temperature at sliding contacts". Proceedings of the royal Society of New South Wales 76 (1942) 203-224.

[6] Laraqi, N., "An exact explicit analytical solution for steady-state temperature of a half space subjected to a circular moving heat source". ASME Journal of Tribology 125 (2003) 859-862.

[7] Hocine, A., Alilat, N., Bauzin, J. G., "Thermal behaviour of a rotating disk subjected to discrete surface heat sources". C.R. Mechanic 337 (8)(2009) 616-620.

[8] Baïri, A., Garcia-de-Maria, J.M., Laraqi, N., "Effect of thickness and thermal properties of film on the thermal behavior of moving rough interfaces". European Physical Journal - Applied Physic 26 (1) (2004) $29-34$.

[9] Ali, Y.M., Zhang L.C., 2005, "Relativistic moving heat source", Int. J. Heat Mass Transfer, 48, pp. 2741-2758.

[10] Bai, D. and Brandt, A., 1987, "Local Mesh refinement MultiLevel Techniques," SIAM J. of Sci. Stat. Comp., 8, pp.109-134.

[11] Rabi, J. A., de Lemos, M. J. S., 2001, "The effects of Peclet number and cycling strategy on multigrid numerical solutions of convective-conductive problems," Appl. Math. Comput. 124. 215-226.

[12] Johnson, K.L., (2004): Contact Mechanics, Cambridge University Press 377-380.

[13] Jaeger, J.C. (1942). Moving sources of heat and temperature at sliding contacts, Proceedings, Royal Society, NSW, 56, 203, [378].

[14] Carslaw, H.S. and Jaeger, J.C. (1959). Conduction of heat in solids, 2nd Ed., Oxford: Clarendon, $[375,376]$.

[15] Liu, S., Lannou, S., Wang, Q., Keer, L. 2004, "'Solutions for Temperature Rise in Stationnary/Moving Bodies Caused by Surface Heating With Surface Convection"' ASME. vol.126, [776-785].

[16] Brandt, A., Multigrid techniques : 1984 guide with applications to fluid dynamics. Gesellschaft fur Mathematic und Datenverarbeitung. MBH BOHN; 1984. p. 176.

[17] Venner C, Lubrecht AA. Multilevel methods in lubrication. Amsterdam: Elsevier; 2000 ISBN 0-44450503-2

[18] Gao, J., Lee, S.C., Ai, C., Nixon, H., 2000, An FFT-based transient flash temperature model for general three-dimensional rough surface contact. ASME Journal pf tribology, vol.122, p. 519-523.

[19] Yu, Y., Farris, T.N., 1997, FFT Thermoelastic solutions for moving heat source. ASME Journal of tribology, vol.119, p.156-162.

[20] Blok, H., The flash temperature concept, Wear 6, 483-494 (1963).

[21] Blok, H., Theoretical Study of the Temperature Rise of Surfaces of Actual Contact Under Oiliness Lubricating Conditions, General Discussion on Lubrication, Inst. Mech. Engrs, London, Vol.2, 1937, pp.222235 .

[22] Archard, J.F., The temperature of Rubbing Surfaces, Wear, Vol.2, 1958/59, pp.438-455.

[23] Laraqi, N., Thermal Constriction Phenomenon in Sliding Contact, Int.J. Heat Mass Transfer, Vol.39, No. 17, 1996, pp.3717-3724. 